\title{
OP16
}

\section{LED SIMULATORS FOR THE REPRODUCTION OF THE NEW CIE STANDARD LED SOURCES}

\author{
Xi Lv et al. \\ DOI 10.25039/x46.2019.OP16 \\ from \\ CIE x046:2019 \\ Proceedings \\ of the \\ 29th CIE SESSION \\ Washington D.C., USA, June 14 - 22, 2019 \\ (DOI 10.25039/x46.2019)
}

The paper has been presented at the 29th CIE Session, Washington D.C., USA, June 14-22, 2019. It has not been peer-reviewed by CIE.

(c) CIE 2019

All rights reserved. Unless otherwise specified, no part of this publication may be reproduced or utilized in any form or by any means, electronic or mechanical, including photocopying and microfilm, without permission in writing from CIE Central Bureau at the address below. Any mention of organizations or products does not imply endorsement by the CIE.

This paper is made available open access for individual use. However, in all other cases all rights are reserved unless explicit permission is sought from and given by the $\mathrm{CIE}$.

CIE Central Bureau

Babenbergerstrasse 9

A-1010 Vienna

Austria

Tel.: +4317143187

e-mail: ciecb@cie.co.at

www.cie.co.at 


\title{
LED SIMULATORS FOR THE REPRODUCTION OF THE NEW CIE STANDARD LED SOURCES
}

\author{
Lv, X. ${ }^{1}$, Luo, M.R. ${ }^{1,2}$ \\ ${ }^{1}$ State Key Laboratory of Modern Optical Instrumentation, Zhejiang University, Hangzhou, CHINA \\ 2 School of Design, University of Leeds, Leeds LS2 9JT, UNITED KINGDOM
}

m.r.luo@zju.edu.cn

DOI $10.25039 / \times 46.2019 .0 P 16$

\begin{abstract}
The LED sources have been developed rapidly in recent years, but there has been no standard spectral power distribution (SPD) for LED sources and the typical illuminants used for colour specification are still CIE D65, F11 and A. LED sources have many advantages and the recent CIE publication 15.4 recommended the standard SPD of LED sources for colour specification. In this study, the LED sources based on spectrum tunable multi-LED systems were made to closely match CIE LED SPDs and the quality of LED sources was assessed through a colour difference experiment. Meanwhile, different formulae were used to evaluate LED sources. The visual results under the simulated sources gave close agreement to those under the CIE LED illuminants.
\end{abstract}

Keywords: Spectral Power Distributions (SPD), CIE LED SPD, lighting quality

\section{Introduction}

The LED technology has been progressed rapidly and greatly changed the lightning industry. Conventional sources are gradually being replaced by LED sources. This could produce problems for the users. For example, shop owners could or have to use LED lamps to illuminate their shops but the colour specification of their products, such as textile cloth, furniture, etc are still based on CIE D65, F11 and A illuminants. They have no idea how close their colour appearance agreeing with those of CIE illuminants. Also, the performance from different lamp manufacturers of the same specification may vary greatly. Thanks to the recent CIE publication $15.4^{[1]}$. We now have the standard spectral power distributions (SPD) of LED sources for colour specification.

On the other hand, by following the CIE publication, many standard LED sources are required for visual inspection. Of course, one way to achieve this is to make single LED to closely match its SPD. Another way to achieve this is to produce SPD match based on spectrum tunable multiLED systems. This work describes the performance of a CIE standard LED simulators.

\section{Methods}

\subsection{Light settings}

Our earlier study ${ }^{2]}$ (Gu and Luo, 2017) verified that a muti-channel LED illumination system, gave very similar visual colour differences as those predicted by CIE D65 and A illuminants. Similar strategy is used here. This study included four real LED light sources $(3000,4000,5000$, $6500 \mathrm{~K})$ which matched closely to the newly published CIE LED SPD. The light sources used here was a multi-channel LED illumination system, LEDCube supplied by the Thouslite Ltd. It was composed of 11 narrow-band LEDs and 3 white phosphor LEDs. It was controlled by a software which is used to adjust various lighting quality parameters. 


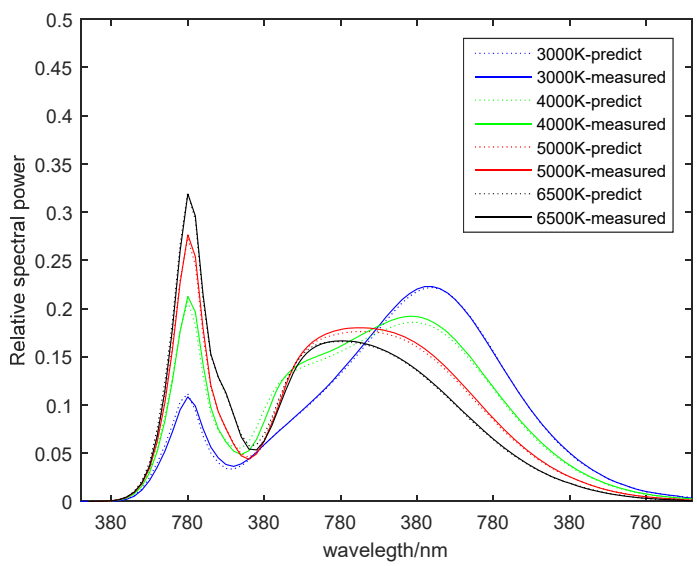

(a)

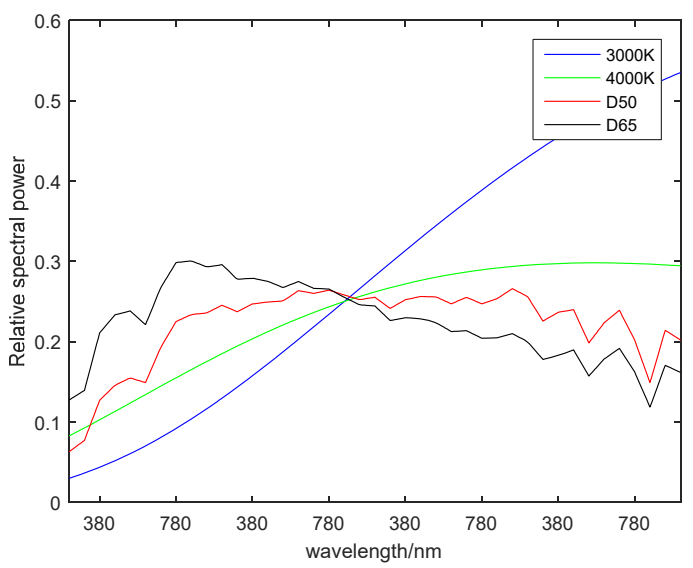

(b)

Figure 1 - SPDs of (a) CIE LED and real LED light sources (predicted ones correspond to CIE LED, measured ones correspond to real LED light sources) and (b) CIE illuminants.

Figure 1 shows the spectral power distribution (SPD) of newly published CIE LED, the real LED light sources and CIE conventional illuminants. It can be seen that all real LED light sources had a close match to the SPD of CIE LED (Figure 1a) and they are very different from the CIE illuminants (Figure 1b).

Table 1 - The colourimetric characteristics of the light settings.

\begin{tabular}{|l|c|c|c|c|c|c|c|}
\hline \multicolumn{2}{|c|}{ LED } & CCT & Lux & $\mathrm{R}_{a}$ & $\mathrm{D}_{u v}$ & RMSE & $\mathrm{MI}$ \\
\hline \multirow{3}{*}{$3000 \mathrm{~K}$} & Predict & 3000 & 3000 & 84 & -0.0035 & $0.9 \%$ & - \\
\cline { 2 - 8 } & Measured & 2812 & 3021 & 83 & -0.0055 & $7.7 \%$ & - \\
\hline \multirow{3}{*}{$4000 \mathrm{~K}$} & Predict & 4000 & 3000 & 87 & -0.0006 & $0.5 \%$ & - \\
\cline { 2 - 8 } & Measured & 4033 & 3010 & 85 & -0.0016 & $6.0 \%$ & - \\
\hline \multirow{3}{*}{$5000 \mathrm{~K}$} & Predict & 5000 & 3000 & 87 & 0.0006 & $0.5 \%$ & 0.27 \\
\cline { 2 - 8 } & Measured & 5011 & 3023 & 78 & 0.0010 & $7.4 \%$ & 0.28 \\
\hline $6500 \mathrm{~K}$ & Predicrt & 6500 & 3000 & 88 & 0.0001 & $0.7 \%$ & 0.28 \\
\cline { 2 - 7 } & Measured & 6580 & 3008 & 79 & 0.0009 & $7.7 \%$ & 0.30 \\
\hline
\end{tabular}

Table 1 shows the colorimetric characteristics of CIE LED and the real LED light settings. The predict data was obtained by fitting the CIE SPDs with the LEDCube system. The root mean square values of LED light settings' SPD were in \% agree with those in the table (0-1 scale), respectively, indicating a quite good match to those of CIE LED standards. The differences in colour rendering index $\left(\mathrm{R}_{a}\right)$, deviation in uv chromaticity relative to the standard illuminant $\left(\mathrm{D}_{u v}\right)$, Illuminance and CCT between the simulated and CIE LED sources were small, i.e. within the tolerances of $\pm 2, \pm 0.001, \pm 50 \mathrm{Lux}$ and $\pm 50 \mathrm{~K}$, respectively. The other metric is metamerism index $(\mathrm{MI})^{[3]}$. In practice, mean colour difference from the 5 virtual metamers calculated under a reference daylight illuminant and a simulator is less than 0.25 and $0.5 \Delta E^{*}$ ab units, the simulator classified as Categories $A$ and $B$ respectively will be considered to be highly satisfactory. It can be seen that under $5000 \mathrm{~K}$ and $6500 \mathrm{~K}$, all the mean $\Delta E^{*}{ }_{a b}$ are close to 0.25 and all had a Category B.

\subsection{Samples}

The visual experiment used the same thirty metametric sample pairs which were produced by dyeing plain wool serge with acid dyes. These samples were prepared by Kuo and Luo ${ }^{[5,6]}$ to investigate the quality of light sources and to evaluate different metamerim indicies.

A JETI Specbos 1211uv tele-spectroradiometer (TSR) was used for measuring samples with an illumination: viewing geometry of $0^{\circ}: 45^{\circ}$. Figure 2 shows the distribution of the 30 metametirc sample pairs in CIELAB colour space under different CIE illuminants, in which vectors represent the colour difference between pairs. It shows a large coverage of the colour gamut. 
The metamers were prepared to have a close visual match under $6500 \mathrm{~K}$ (D65) and a very large mismatch under $3000 \mathrm{~K}$. Figure 2 shows that the test metamers exhibit larger colour differences, or colour shift in the green- red or $a^{*}$ direction under a lower correlated colour temperature (CCT), which also indicates these metamers to have a higher degree of metamerism in lower CCT than higher CCT.
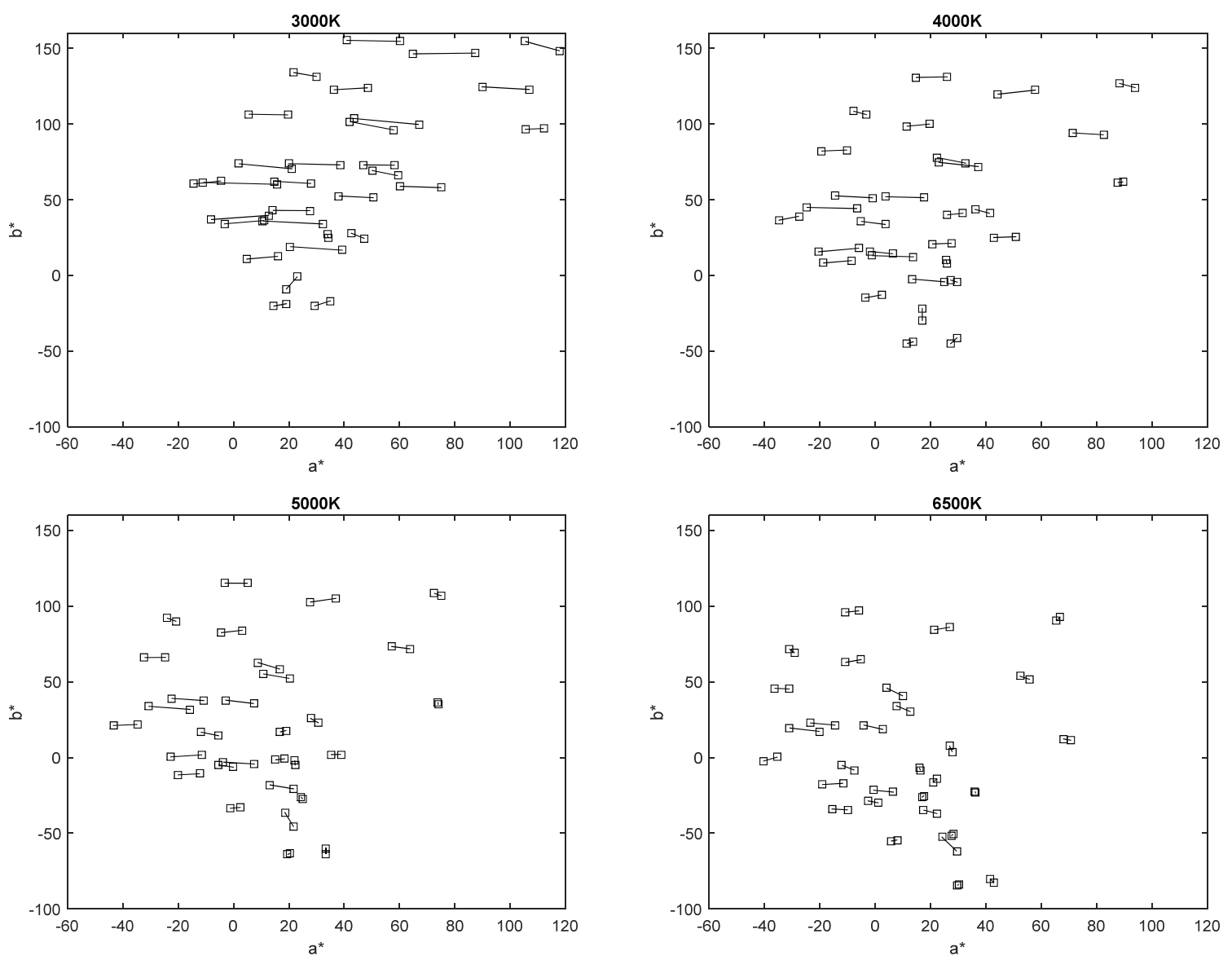

Figure 2 - Distribution of the thirty metamers in CIELAB colour space under four CIE LED illuminants.

\subsection{Observers}

Twenty observers (ten male and ten female observers) aged between 21 and 26 with an average age of 23 participated the psychophysical experiment. All of them had normal colour vision tested by Ishihara colour vision test.

\subsection{Experimental}

Observers viewed samples against a gray-background in a lighting box (shows in Figure 3 ) and the viewing geometry was controlled at $0^{\circ}: 45^{\circ}$. The visual experiment was done to assess colour difference using the widely used gray scale method which is based on ISO $105^{[4]}$ used a 9grade scale (shows in Figure 3) from 1 to 5 within an interval of 0.5 , in which Grade 5 and Grade 1 exhibits zero and very large visual colour differences, respectively.

The experiment was carried out in a dark room and observers passed the Ishihara test before the experiment. At the beginning of the experiment, observers adapt to the gray background illuminated by one LED simulator from the four light settings which were shown in Table 1. During the experiment, observers need to accomplish visual assessments according to colour differences between pairs of samples. The test metamers were placed on top of the gray scale in the lighting box. The gray scale method was based on ISO 105: Part A02 Gray Scale for Assessing Change in Colour. It shows a reduction of colour difference with an increase of grade ratings. Observers assessed pairs of samples using the gray scale and were encouraged to 
give intermediate ratings. In the assessment, Grade 1 means the largest colour difference and Grade 5 represents no colour difference. Observers were encouraged to give a rating in fraction, i.e. 2.8 means the difference is close to that between the standard and Grade 3.
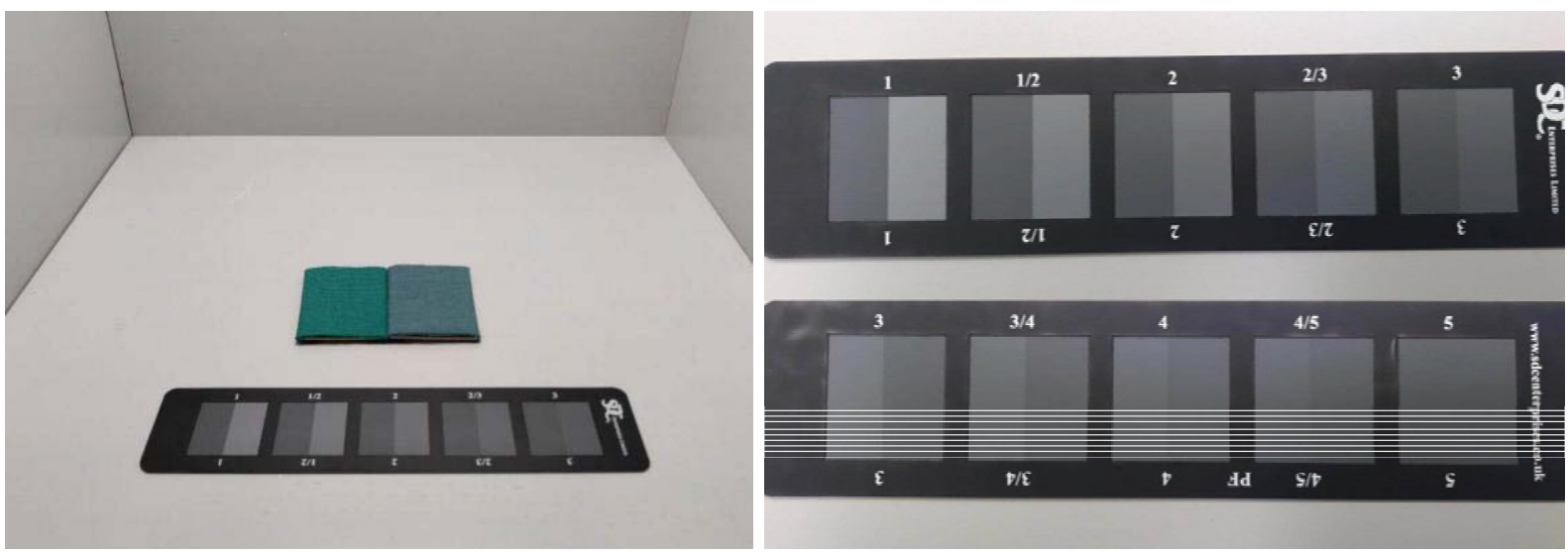

Figure 3 - Viewing condition of colour difference experiment and two sides of the gray grade card.

Totally, the experiment took about $1 \mathrm{~h}$ for each observer and 2,400 assessments were accumulated, that is, 30 pairs $\times 4$ simulators $\times 20$ observers.

\section{Results}

The STRESS is a statistical measure widely used in colour research field to indicate the disagreement between two sets of data. A value of zero means a perfect agreement between them and it is in a scale of $0-100$. The results showed very small STRESS values The STRESS value calculated from equation (1) was used to indicate the disagreement between $A$ and $B$ datasets.

$$
\text { STRESS }=\left(\frac{\sum_{i=1}^{n}\left(A_{i}-F B_{i}\right)^{2}}{\sum_{i=1}^{n} F^{2} B_{i}^{2}}\right)^{1 / 2} \times 100
$$

and $F=\sum_{i=1}^{n} A_{i}^{2} / \sum_{i=1}^{n} A_{i} B_{i}$

where $\mathrm{n}$ is the number of samples and $\mathrm{F}$ is a scaling factor to adjust $\mathrm{A}$ and $\mathrm{B}$ data sets on to the same scale. The percent STRESS values are always between 0 and 100 . Values of STRESS near to zero indicate better agreement between two sets of data.

\subsection{Colour Difference Assessment}

The raw experimental data reported was recorded as a rating of 1 to 5 . The colour difference of each grade against Grade 5 was calculated in terms of CIELAB unit. A polynomial equation was fitted to predict CIELAB colour difference from Grade. The equation (Eq.2) was used to transform each observer's raw data to visual difference $(\Delta V)$, in CIELAB unit.

$$
\Delta V=0.052 G^{4}-0.926 G^{3}+6.238 G^{2}-19.9 G+27.03
$$




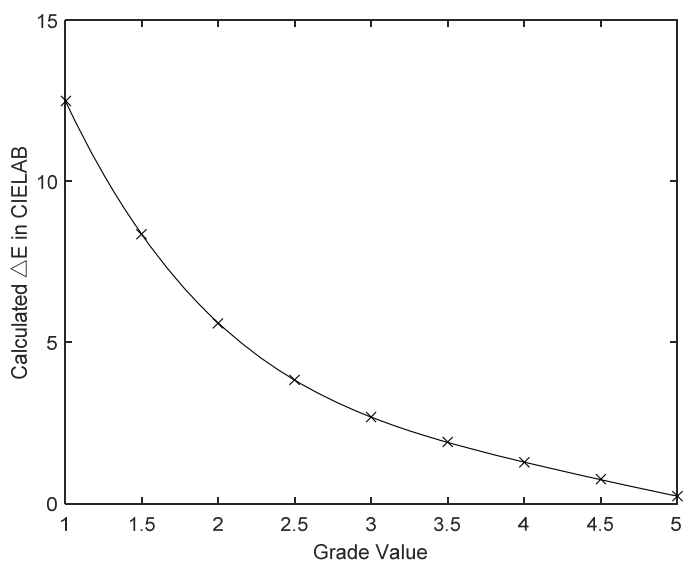

Figure 4 - The relationship between grade values and calculated CIELAB colour differences.

The reliability of colour difference assessment was evaluated in terms of inter-observer variability quantified by the STRESS values, where the two data sets are the $\Delta V$ results of each observer and the $\Delta V$ results of the mean observer, respectively. Table 2 shows the interobserver variability of colour difference assessment.

Table 2 - Inter-observer variability of colour difference assessment in terms of STRESS

\begin{tabular}{|c|c|c|c|c|}
\hline & $3000 \mathrm{~K}$ & $4000 \mathrm{~K}$ & $5000 \mathrm{~K}$ & $6500 \mathrm{~K}$ \\
\hline STRESS & 27 & 29 & 35 & 39 \\
\hline
\end{tabular}

\subsection{Performance of Colour Difference Formulae}

The visual difference results were then used to evaluate the quality of simulators. The STRESS values were calculated between the visual colour differences $(\Delta V)$ and the predicted CIEDE2000 colour differences $(\Delta E)$ of the real and CIE LED illuminants. Note CIEDE2000 formula is recommended. Figure 5 shows the relationship between visual differences and the predicted CIEDE2000 colour differences of the CIE LED illuminants.
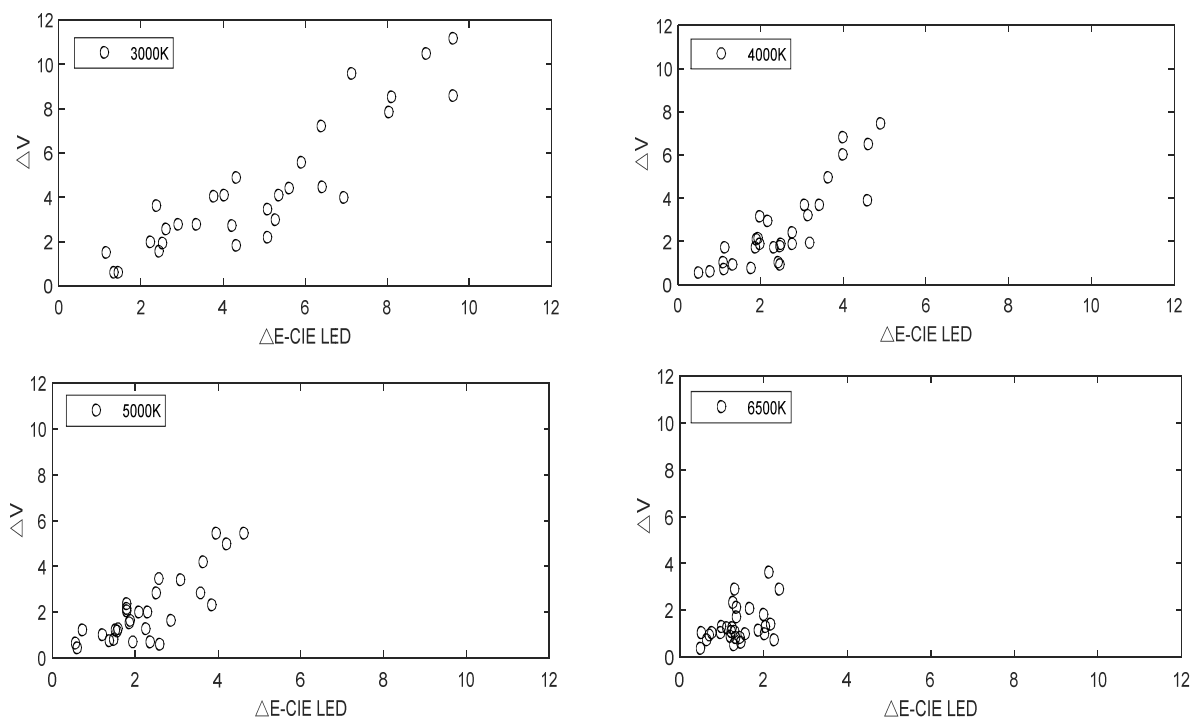

Figure 5 -The predicted CIEDE2000 colour differences plotted against visual differences under the CIE LED illuminants.

Finally, the performance of three colour-difference formulae, CIEDE2000, CIELAB and CAM02UCS were tested to predict visual results from different illuminants in terms of STRESS values. 
Table 3 shows that the real illuminants performed slightly better than the corresponding CIE standard illuminants. The differences were very small, indicating the multiple LED simulator can achieve satisfactory simulation to the standard LED illuminant and can be confidently used for commercial evaluation. However, by replacing the CIE LED to the conventional CIE illuminants, Table 3 shows that the latter all poorly predicted the visual results. This indicates that the new CIE LED sources have quite different property as the conventional CIE standard sources. This strongly suggests that to present objects under LED sources, colour specification must use those new CIE LED illuminants.

Table 3 - Different models' performance in terms of STRESS values.

\begin{tabular}{|c|c|c|c|c|c|c|c|c|c|}
\hline & \multicolumn{3}{|c|}{ CIELAB } & \multicolumn{3}{c|}{ CIEDE2000 } & \multicolumn{3}{c|}{ CAM16-UCS } \\
\hline CCT & $\begin{array}{c}\text { Real } \\
\text { LED }\end{array}$ & $\begin{array}{c}\text { CIE } \\
\text { LED }\end{array}$ & $\begin{array}{c}\text { CIE } \\
\text { illumin- } \\
\text { ants }\end{array}$ & Real & $\begin{array}{c}\text { CIE } \\
\text { LED }\end{array}$ & $\begin{array}{c}\text { CIE } \\
\text { illumin- } \\
\text { ants }\end{array}$ & Real & $\begin{array}{c}\text { CIE } \\
\text { LED }\end{array}$ & $\begin{array}{c}\text { CIE } \\
\text { illumin- } \\
\text { ants }\end{array}$ \\
\hline $3000 \mathrm{~K}$ & 39 & 39 & 41 & 24 & 25 & 41 & 29 & 29 & 32 \\
\hline $4000 \mathrm{~K}$ & 45 & 49 & 52 & 29 & 32 & 52 & 33 & 37 & 42 \\
\hline $5000 \mathrm{~K}$ & 43 & 43 & 63 & 30 & 32 & 62 & 34 & 35 & 58 \\
\hline $6500 \mathrm{~K}$ & 44 & 52 & 55 & 36 & 44 & 53 & 37 & 46 & 54 \\
\hline
\end{tabular}

The results in Table 3 clearly showed that CIEDE2000 performed the best, followed by CAM02UCS slightly worse and CIELAB performs the worst. Comparing the STRESS values between the CIEDE2000 (Table 3) and the inter-observer variability (Table 2), it can be seen the former gave consistently smaller values than those of latter. This means the predictive error from CIEDE2000 is smaller than the uncertainty of visual assessments from a group of 20 observers, indicating an accurate performance. This confirms the results from many other studies, CIEDE2000 outperformed the other formulae. Table 4 also shows that each formula performs substantially consistent under both the real LED light sources and the CIE LED illuminants, and there is also a great similarity between the real LED light sources and their targeted CIE LED illuminants.

\subsection{Close agreement between the real and CIE LED illuminations}

A direct way to show the similarity between the real and CIE LED illumination is to compare the colour differences calculated for each formula. Again, the 3 formulae tested earlier were used. The colour differences of the 30 metamers were calculated between the real light sources and the CIE standard LED. Table 4 shows the similarity between the two illuminants in terms of STRESS. This implies that the real LED sources had a high degree of matching with the CIE LED sources and the test metamers exhibit larger colour differences under a lower correlated colour temperature (CCT) (3000K and 4000K). Figure 6 shows the CIEDE2000 values calculated from the CIE LED against those calculated from the real illuminant. They showed excellent agreement between the real and CIE LED illuminants. This indicates that real illuminant gave a very successful simulation to the CIE LED illuminants.

Table 4 - The agreement of the two sets of illuminants (real LED illuminants and CIE IED illuminants).

\begin{tabular}{|c|c|c|c|c|}
\hline & $3000 \mathrm{~K}$ & $4000 \mathrm{~K}$ & $5000 \mathrm{~K}$ & $6500 \mathrm{~K}$ \\
\hline CIEDE2000 & 6 & 5 & 4 & 15 \\
\hline CAM16-UCS & 5 & 6 & 5 & 14 \\
\hline CIELAB & 4 & 7 & 6 & 13 \\
\hline
\end{tabular}

The STRESS values for all formulae are the largest for $6500 \mathrm{~K}$ illuminants as expected, because evaluating smaller colour differences always has a larger visual disagreement or gives larger colour difference predictive error. Note that the metamers here were prepared to have small colour differences under D65/10 condition. 

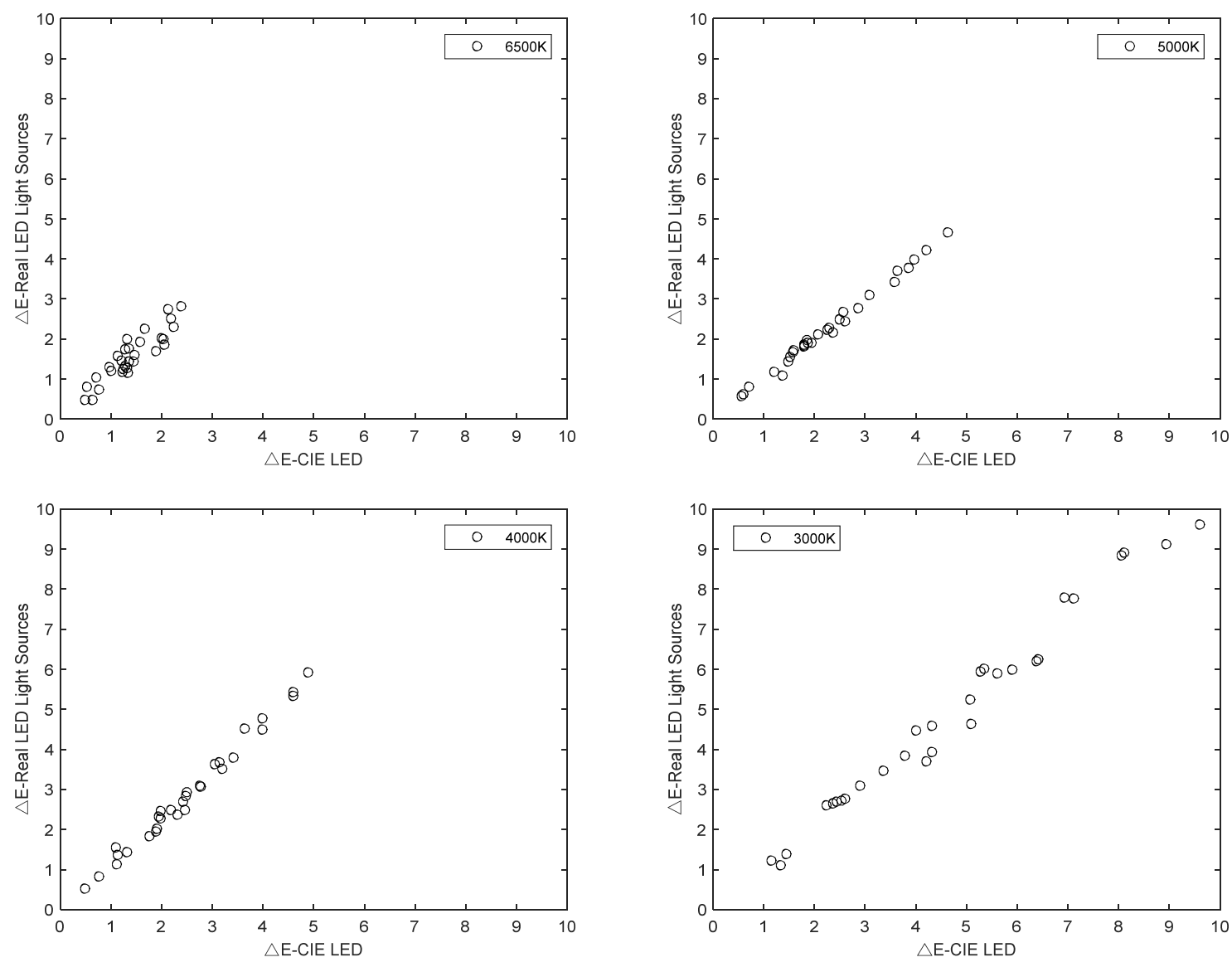

Figure 6 - CIEDE2000 colour differences plotted between the real and CIE LED illuminants.

\section{Conclusions}

An experiment was conducted to test the performance of a spectrum tunable LED system to simulate the newly published CIE LED standard sources. It matches all the colour quality criteria in terms of CCT, $\mathrm{D}_{u v}, \mathrm{R}_{a}$, Lux. The visual experiments were conducted under those real simulated sources. The visual results were used to test different colour difference formulae. The results showed CIEDE2000 giving the most accurate prediction. Finally, the agreement between the real and CIE LED illuminants was verified. It was found a good agreement between the visual results and the colour differences calculated using the real illuminant and between the visual results and the colour differences calculated using the new CIE LED standard illuminants. However, using the new data to test the conventional standard CIE illuminants, they predicted poorly to the visual results. This implies for applying LED sources, the newly published CIE LED illuminants should be used for color specification.

\section{Acknowledgement}

The work is supported by National Natural Science Foundation of China (Grant number: 61775190)

\section{References}

[1] CIE 15.4: 2018 Colourimetry, Vienna: Commission Intermationale de I'Eclairage; 2018.

[2] Gu, H. T., Pointer, M. R., Liu, X. Y., \& Ronnier Luo, M. 2017. Quantifying the Suitability of CIE D50 and A Simulators Based on LED Light Sources. Colour Research \& Application, 42(4), 408-418. doi:10.1002/col.22098 
[3] CIE 51.2: 1995 A Method for Assessing the Quality of Daylight Simulators for Colourimetry, Vienna: Commission Intermationale de l'Eclairage; 1999.

[4] ISO 105: 1996: Textiles - Tests for Colour Fastness - Part A02: Grey Scale for Assessing Change in Colour: Geneva: International Organization for Standardization; 1996.

[5] W. G. Kuo and M. R. Luo, Methods for quantifying metamerism. Part I - Visual assessment, J. Soc. Dyers. Col. 112 (1996) 312-320.

[6] W. G. Kuo and M. R. Luo, Methods for quantifying metamerism. Part II - Instrumental assessment, J. Soc. Dyers. Col. 112 (1996) 354-360. 